\title{
A Continuous Adiabatic Demagnetization Refrigerator for Use with Mechanical Coolers
}

\author{
P. Shirron', N. Abbondante', E. Canavan', M. DiPirro', M. Grabowski², M. \\ Hirsch $^{2}$, M. Jackson' ${ }^{1}$ and J. Tuttle ${ }^{1}$ \\ 'NASA/Goddard Space Flight Center \\ Code 552 \\ Greenbelt, MD, USA 20771 \\ ${ }^{2}$ Worcester Polytechnic Institute/Department of Physics \\ Worcester, MA, USA 01609
}

\begin{abstract}
We have begun developing an adiabatic demagnetization refrigerator (ADR) which can produce continuous cooling at temperatures of $50 \mathrm{mK}$ or lower, with high cooling power (goal of $10 \mu \mathrm{W}$ ). The design uses multiple stages to cascade heat from a continuously-cooled stage up to a heat sink. The serial arrangement makes it possible to add stages to extend the operating range to lower temperature, or to raise the heat rejection temperature. Compared to conventional single-shot ADRs, this system achieves higher cooling power per unit mass and is able to reject its heat at a more uniform rate. For operation with a mechanical cryocooler, this latter feature stabilizes the heat sink temperature and allows both the ADR and cryocooler to operate more efficiently. The ADR is being designed to operate with a heat sink as warm as 10-12 K to make it compatible with a wide variety of mechanical coolers as part of a versatile, cryogen-free low temperature cooling system. A two-stage system has been constructed and a proof-of-principle demonstration was conducted at $100 \mathrm{mK}$. Details of the design and test results, as well as the direction of future work, are discussed.
\end{abstract}

\section{INTRODUCTION}

Adiabatic demagnetization of a magnetocaloric material (that is, a material that exhibits a marked change in entropy due to an applied magnetic field) is a decades old method for producing sub Kelvin temperatures in the laboratory. Recently adiabatic demagnetization refrigerators (ADRs) have been developed for space flight [refA,B,C] to cool infrared and x-ray detectors to below $0.1 \mathrm{~K}$. More low temperature detectors are planned for future space flights including superconducting tunnel junctions coupled to rf single electron transistors[refD], transition edge sensors[refE], spiderweb bolometers[refF] to measure $x$-ray, ultraviolet, infrared, and submillimeter radiation. Refrigeration is needed not only to cool detectors to sub Kelvin temperatures, but to cool the detector surroundings, or 


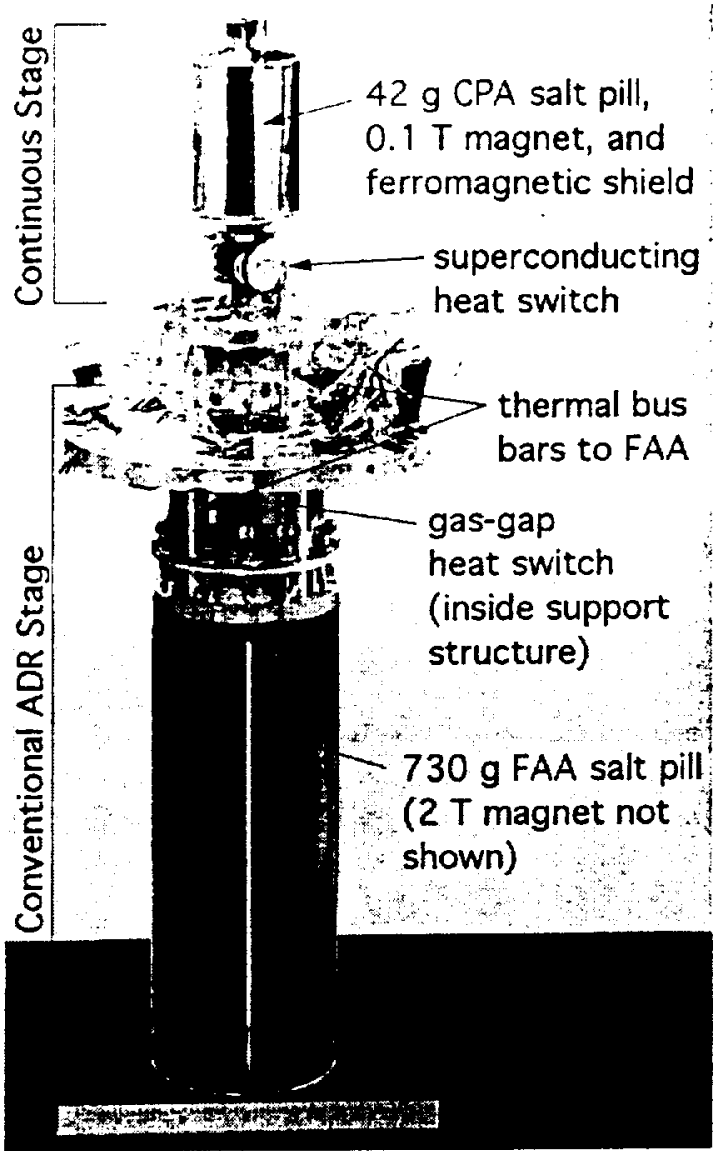

Figure 1. Two-stage ADR developed to demonstrate continuous low temperature cooling.

\section{DEMONSTRATION OF CONTINLOLS COOLING}

Since the characteristics of the continuous stage, including thermodynamic efficiency, duration of the recycling operation, parasitic heat flow through the heat switch, etc., will have the single largest impact on the design of a full-up. ADR, our effort so far has been devoted to conducting a demonstration of continuous cooling at low temperature. The primary goal was to characterize the extent to which one ADR stage could be operated at a constant temperature while heat was periodically offloaded to a second stage. This required developing both a "continuous" ADR stage and the electronics and control algorithms for automating the recycling process. It also provided an opportunity to probe such details as magnetic interactions between stages and which factors were most important for maintaining high thermodynamic efficiency in the process. The demonstration was performed using two stages connected in series as shown in Fig. 1. Test results are now being used to iterate the design of the continuous stage and to establish a design of a system capable of operating between $50 \mathrm{mK}$ and $10 \mathrm{~K}$. Details of this work are provided in the next section.

The continuous stage consists of a $+2 \mathrm{~g}$ CPA salt pill. a $0.1 \mathrm{~T}$ magnet, and a thin layer of magnetic shielding connected to a much larger stage (consisting of a $730 \mathrm{~g} \mathrm{FAA}$ salt pill and a $2 \mathrm{~T}$ magnet) through a superconducting indium heat switch. The FA.t stage has enough cooling capacity to initially cool the CPA to its operating point then to act as its heat sink for several days without needing to be recycled. The mass of CP.A was less than the container was designed to hold ( $52 \mathrm{~g}$ ) due to problems encountered when growing the salt. but it is enough to give the salt pill a hold time of -1 hour with a heat load of $10 \mu W$ at $10.15 \Sigma-1) .1 \mathrm{~K}$.

The configuration of the continuous stuge is unusual in that the magnet is physically and thermally anchored to the CPA salt pill. Ordinarily the magnet is attached to the heat sink and the salt pill is suspended inside it. However. in vur case the arrangement simplities the mechanical design 


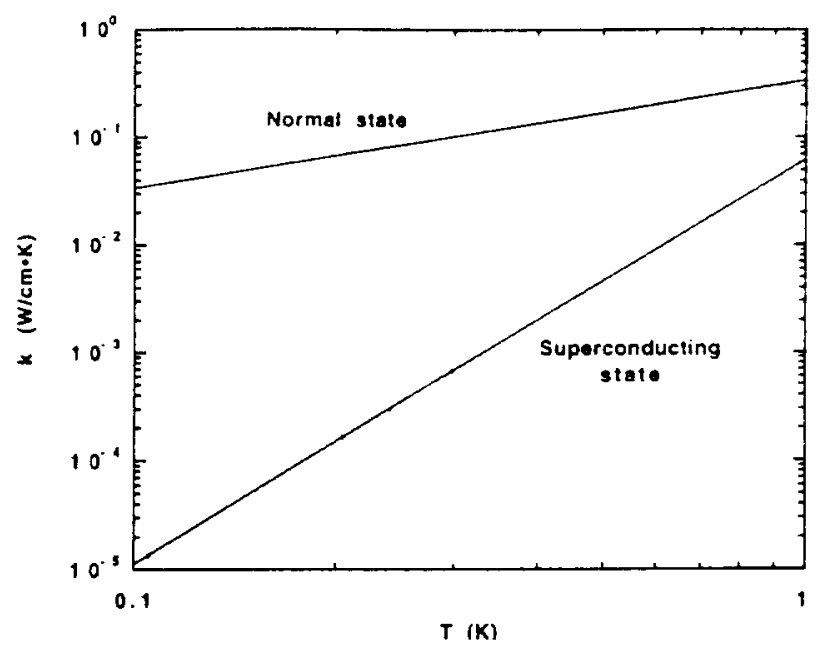

Figure 3. Thermal conductivity of the indium heat switch in the normal and superconducting states.

and copper end pieces. The switch is activated by a pair of superconducting Helmholtz coils that produce a field perpendicular to the direction of heat flow. This prevents trapped flux in the indium from thermally shorting the switch in the off state.

Measurements of the on- and off-state thermal conductivity of the switch are shown in Fig. 3. The strong temperature dependence of the off conductance limits the temperature at which the FAA can be stationed when the CPA is not being recycled to less than about $0.3 \mathrm{~K}$. At that temperature, the $3 \mu \mathrm{W}$ of heat flowing to the colder CPA stage is a substantial fraction of its target cooling power of 10 $\mu W$.

\section{Continuous Cooling Tests}

Generally, each test consisted of initially recycling the FAA and demagnetizing to $0.1 \mathrm{~K}$ with the superconducting heat switch turned on. Once the CPA equilibrates, a temperature controller is activated to maintain the thermal strap that is connected to the load at $0.1 \mathrm{~K}$. This strap is sandwiched between the superconducting heat switch and the CPA salt pill. Temperature regulation of both the FAA and the strap is achieved with digital PID feedback loops using low noise power supplies to regulate the FAA and CPA magnet currents. While our eventual goal is to cool the load to $50 \mathrm{mK}$ or below, the higher ordering temperature of FAA $\left(\mathrm{T}_{\mathrm{c}}-35 \mathrm{mK}\right)$ prevents us from having sufficient cooling power at $50 \mathrm{mK}$. For this reason, all tests have been conducted at $100 \mathrm{mK}$.

With the base temperature established, a simple sequencing routine takes over to automatically recycle the CPA when its cooling capacity is nearly exhausted. Typical results are shown in Fig. 4a. The characteristic sawtooth pattern in CPA current results from alternately magnetizing the CPA as it transfers heat to the FAA stage, then demagnetizing the CPA as it actively cools its load. The details of this sequence are as follows:

1. Throughout most of the cycle, the CPA is thermally isolated from the FAA (heat switch is off) and is receiving heat from its load and parasitic sources. To maintain constant temperature, the CPA is slowly demagnetized. Meanwhile, the FAA is stationed at a higher temperature to absorb its parasitic heat loads more efficiently.

2. When the current approaches zero (or falls below a preset threshold), the recycling process begins:

2a. The FAA is cycled down to $0.1 \mathrm{~K}$

2b. The superconducting heat switch is turned on. For this event. it is critical for the FAA and CPA to be at the same temperature to prevent a sudden change in the heat flow to/from the CPA. 
In principle, though, there is no intrinsic limit to the stability that can be achieved. and we are making several changes that will improve the situation. The first is in the construction of a new CPA salt pill with its high conductance thermal bus. The bus is also gold-plated to reduce the joint resistance at the bolted interface (an oversight on the current salt pill). The second change is to increase the bandwidth of the temperature controller. This will be done either using a second computer dedicated to the task, or an analog controller. Further improvements may result from limiting the rates at which the FAA's temperature is adjusted to a time constant longer than the bandwidth of the controller.

\section{Thermodynamic Efficiency}

The thermodynamic efficiency of the continuous stage was a particular concern because of several compromises that were made in the design. Mostly these had to do with increasing the amount of copper used in the heat switch and thermal bus in order to increase the thermal conductance of each bolted joint and of the thermal bus as a whole. The main concern was over eddy current heating in the copper as the local magnetic fields were varied. This could have been particularly problematic since the inductance of the CPA magnet is small and voltage noise at the power supply could lead to large instantaneous ramp rates. On the other hand, we expected an improvement in efficiency over conventional ADR systems because we could use a much longer fraction of time to perform the recycle operation and therefore keep thermal gradients in the system to a smaller fraction of absolute temperature. This of course depends on the fraction of time used for recycling. For these measurements, the fraction was the same $20 \%$ which we project will be typical of a full-up continuous ADR.

We have characterized the efficiency of the continuous stage at two levels. The first quantifies the performance of the salt pill itself in terms of the amount of heat it can absorb during the demagnetization portion of the cycle compared to the amount of heat that is rejected during as it is magnetized. The second, which is more appropriate for comparing the present ADR to other refrigerators, was to determine the heat rejected to the FAA heat sink compared to that absorbed during the cooling portion of the cycle.

The measurement of salt pill efficiency was made by comparing magnetization (and demagnetization) rates when only nominal parasitic heat loads were present and again when an additional heat load of 1 or $2 \mu \mathrm{W}$ was applied. The resulting figure of $94 \%$ is almost completely accounted for by the $4 \mathrm{mK}$ change in the CPA's temperature (at $100 \mathrm{mK}$ ) as it transitioned from absorbing to rejecting heat. This leads us to conclude that eddy current heating in the heat switch and salt pill is not a significant concern.

Finally, by measuring the amount of heat rejected to the FAA during recycling, we arrive at a figure of $88 \%$ for the efficiency of the continuous stage operating at $0.1 \mathrm{~K}$. For comparable cooling power at lower temperature, we expect lower efficiency due to larger thermal gradients, particularly at the salt/thermal bus boundary, which will be an even larger fraction of absolute temperature. These will be offset to some extent with recent improvements in the salt pill construction, so we expect to maintain an efficiency at least in the $70-80 \%$ range. For the higher temperature stages in a multi-stage system. we expect to achieve efficiencies in the $80-90 \%$ range, so that a full scale system operating between $50 \mathrm{mK}$ and $10 \mathrm{~K}$ could achieve an overall efficiency of $35-50 \%$.

\section{FUTURE WORK}

The two primary directions for future work are to continue developing the components needed for a continuous cooling demonstration at $50 \mathrm{mK}$, and to begin work on materials and components for the high temperature stages $(1-10 \mathrm{~K})$. For the low temperature work, we need to develop another $\mathrm{CPA}$ stage that can act as the heat sink for the existing continuous stage. Using CPA will allow us to access temperature down to as low as $30 \mathrm{mK}$. We also working on a design for a new liquid-gap heat switch which will connect this CPA stage to the existing FAA stage. The characteristics of this switch 
D. R.J. Schoelkopf, S.H. Moseley, C.M. Stahle, P. Wahlgren and P. Delsing, A concept for a submillimeter-wave single-photon counter, submitted for publication in IEEE Transactions on Applied Superconductivity, vol. 9 (1999).

E. K.D. Irwin, G.C. Hilton, D.A. Wollman, and J.M. Martinis, X-ray detection using a superconducting transition-edge sensor microcalorimeter with electrothermal feedback, Appl. Phys. Lett. 69:1945 (1996).

F. J.J. Bock, et al., Silicon nitride micromesh bolometer arrays for SPIRE, Proc. SPIE vol. 3357: 297-304 (1998).

G. S. Breon, et al., The XRS low temperature cryogenic system ground performance tests results, Cryogenics 39: 677(1999).

H. P.J. Shirron, E.R. Canavan, M.J. DiPirro, J.G. Tuttle, and C.J. Yeager, A multi-stage continuousduty adiabatic demagnetization refrigerator, $A d v$. Cryo. Eng. 45, to be published.

I. I. Numazawa, GGG/DGG mixtures

J. J. Shull, GGIG 